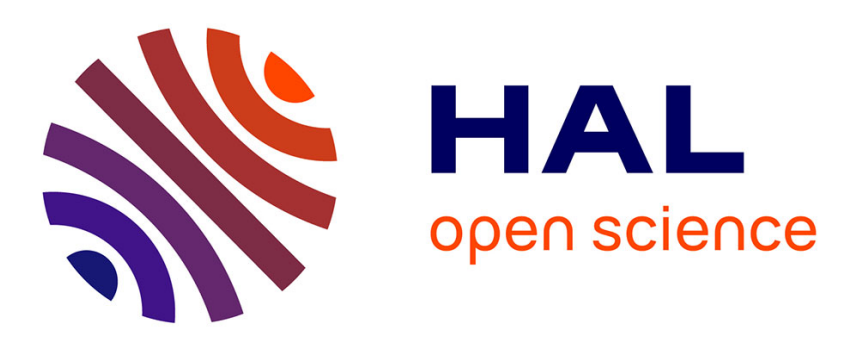

\title{
Informativity: how to get just sufficiently rich for the Identification of MISO FIR Systems with Multisine Excitation?
}

Kévin Colin, Xavier Bombois, Laurent Bako, Federico Morelli

\section{- To cite this version:}

Kévin Colin, Xavier Bombois, Laurent Bako, Federico Morelli. Informativity: how to get just sufficiently rich for the Identification of MISO FIR Systems with Multisine Excitation?. 2019. hal02070880v2

\section{HAL Id: hal-02070880 \\ https://hal.science/hal-02070880v2}

Preprint submitted on 20 Jun 2019

HAL is a multi-disciplinary open access archive for the deposit and dissemination of scientific research documents, whether they are published or not. The documents may come from teaching and research institutions in France or abroad, or from public or private research centers.
L'archive ouverte pluridisciplinaire HAL, est destinée au dépôt et à la diffusion de documents scientifiques de niveau recherche, publiés ou non, émanant des établissements d'enseignement et de recherche français ou étrangers, des laboratoires publics ou privés. 


\title{
Informativity: how to get just sufficiently rich for the Identification of MISO FIR Systems with Multisine Excitation?
}

\author{
Kevin Colin ${ }^{1}$, Xavier Bombois ${ }^{1}$, Laurent Bako $^{1}$ and Federico Morelli ${ }^{1 *}$
}

June 20, 2019

This technical report presents a pre-print version of a paper that has been submitted and accepted for the proceedings of the European Control Conference 2019.

\begin{abstract}
In Prediction Error Identification, the consistency of the identified parameter vector is only guaranteed if the data are informative enough i.e. if the excitation signal is sufficiently rich. For single-input single-output systems, one can verify whether a given excitation is sufficiently rich for a system based on the number of frequencies at which its power spectrum is nonzero. The extension of this criterion to multivariate systems is not straightforward. In the literature, one has proposed criteria based on the number of frequencies at which the power spectrum matrix of the excitation signal is strictly positive definite. However, this criterion is too restrictive as it does not cover the case of multisine excitations, while it is well known that such excitation signals can lead to consistent estimates. This paper proposes less restrictive conditions for the consistency of the identified parameter vector when FIR multiple-inputs single-output systems have to be identified with multisine signals in the open loop configuration.
\end{abstract}

\footnotetext{
*1 Laboratoire Ampere, UMR CNRS 5005, Ecole Centrale de Lyon, Université de Lyon, Ecully, France,
} kevin.colin@ec-lyon.fr 


\section{Introduction}

In System Identification, there are two main conditions to get a consistent estimate of the parameter vector: the identifiability of the model structure and the data informativity with respect to (w.r.t.) this model structure [1]. These concepts have been well studied for single-input singleoutput (SISO) model structures both in the open loop and the closed loop configurations [2]. In the open loop case, the data informativity is linked to the input richness. In [1] and [3], there is a simple criterion to evaluate the input richness: it is the number of frequencies at which its power spectrum is nonzero. For the multivariate case, the richness definition of an input vector can be extended by considering the number of frequencies at which the input power spectrum matrix is positive definite [3]. However, this criterion does not cover the multisine case as the input power spectrum matrix degenerates at all frequencies. In this paper, we propose less restrictive conditions for multiple-inputs single-output (MISO) finite impulse response (FIR) systems in open loop with multisine inputs.

For MISO FIR structure, the identifiability is verified: the data informativity w.r.t. this model structure is equivalent to the consistency of the estimate [2]. In open loop, the data informativity is equivalent to the persistency of the input regressor derived from the MISO FIR model structure [2]. Thus, the problem considered in this paper is the development of necessary and sufficient conditions on the input vector such that the input regressor derived from the MISO FIR structure is persistently exciting. A necessary and sufficient condition for the regressor persistency is the linearly independence of the signals of this regressor [2]. Our research problem becomes the following one: finding sufficient conditions on the input vector such that the signals in this regressor are linearly independent. For multisine inputs, the linearly independence of the signals of the input regressor is equivalent to the left invertibility of a particular matrix which depends, on one hand, on the chosen amplitudes, phase-shifts and frequencies of the multisine and, on the other hand, on the FIR model orders. This simple matrix criterion answers our research problem with multisine inputs.

However this matrix criterion is not enough explicit on how to effectively make a choice of the amplitudes, of the phase-shifts and of the sinusoid number such that this matrix is left invertible or full-column rank (a posteriori criterion). Therefore, we propose additional conditions on the number of sinusoids to use and on the amplitude and phase-shift choices. These conditions are only necessary, but they guide the user in its multisine choices. Finally, we propose two particular multisine choices which always lead to the regressor persistency (and so the estimation consistency) regardless of the amplitude and phase-shift choices.

Notations: For a complex-valued matrix $A, A^{T}$ denotes its transpose and $A^{*}$ its complex conjugate. The notation $\operatorname{diag}\left(a_{1}, \cdots, a_{n}\right)$ denotes the diagonal matrix whose diagonal elements are $a_{1}, \cdots, a_{n}$ (scalars or matrices). The notation $j$ is the complex number such that $j^{2}=-1$. For all nonzero integers $m$, the set $\llbracket 1, m \rrbracket$ is the set of consecutive integers between 1 and $m$, i.e. $\{1,2, \cdots, m\}$. We denote $x(\bullet)$ the function describing the time-domain signal $x(t) \forall t$.

\section{Prediction error of MISO FIR system}

Let $\mathcal{S}$ be a MISO FIR system with $p$ inputs and one output:

$$
\mathcal{S}: y(t)=G_{0}(z) u(t)+e(t)
$$


where $z$ is the forward-shift operator, $G_{0}(z)=\left(G_{0_{1}}(z), \cdots, G_{0_{p}}(z)\right)$ is the transfer function matrix, $u(t)=\left(u_{1}(t) \cdots u_{p}(t)\right)^{T}$ is the input vector of size $p \times 1, y(t)$ is the scalar output and $e(t)$ is a white noise with variance $\sigma_{0}^{2}$. We assume that each $u_{i}(t)$ is a quasistationary process [1] independent of $e(t)$. For the sake of simplicity, all $G_{0_{i}}(z)$ are FIR systems with the the same order $m$ and have a delay equal to $1: G_{0_{i}}(z)=\beta_{i 1} z^{-1}+\cdots+\beta_{i m} z^{-m}$. The system $\mathcal{S}$ in (2.1) is identified within a model structure denoted $M(\theta)=G(z, \theta)$, parametrized by a vector $\theta \in \mathbb{R}^{p m}$, where $G(z, \theta)=\left(G_{1}(z, \theta) \cdots G_{p}(z, \theta)\right)$ and:

$$
G_{i}(z, \theta)=b_{i 1} z^{-1}+\cdots+b_{i m} z^{-m}
$$

All $b_{i k}$ with $i \in \llbracket 1, p \rrbracket$ and $k \in \llbracket 1, m \rrbracket$ are the parameters to be identified. We will denote $\theta_{i}=\left(\begin{array}{lll}b_{i_{1}} & \cdots & b_{i_{m}}\end{array}\right)^{T}$. Therefore we have $\theta^{T}=\left(\begin{array}{lll}\theta_{1}^{T} & \cdots & \theta_{p}^{T}\end{array}\right)$.

We will assume that the model structure $M(\theta)$ is full-order, i.e. $\exists \theta_{0} \in \mathbb{R}^{p m}$ such that $G\left(z, \theta_{0}\right)=$ $G_{0}(z)$. From $M(\theta)$, we define the one-step ahead predictor as follows:

$$
\hat{y}(t, \theta)=G(z, \theta) u(t)=\sum_{i=1}^{p} \phi_{u_{i}}^{T}(t) \theta_{i}
$$

where $\phi_{u_{i}}^{T}(t)=\left(u_{i}(t-1) \cdots u_{i}(t-m)\right)$ is a regressor of the scalar input $u_{i}(t)$. We will call

$$
\phi_{u}(t)=\left(\begin{array}{c}
\phi_{u_{1}}(t) \\
\vdots \\
\phi_{u_{p}}(t)
\end{array}\right)
$$

the input regressor derived from $M(\theta)$. Then (2.3) becomes:

$$
\hat{y}(t, \theta)=\phi_{u}^{T}(t) \theta
$$

We define from (2.5) the prediction error:

$$
\epsilon(t, \theta)=y(t)-\hat{y}(t, \theta)=y(t)-\phi_{u}^{T}(t) \theta
$$

With $N$ input-output data generated from $\mathcal{S}$, we compute the optimal $\theta$, denoted $\hat{\theta}_{N}$ such that it minimizes a least-square cost-function on the prediction error:

$$
\begin{aligned}
\hat{\theta}_{N} & =\arg \min _{\theta} V_{N}(\theta) \\
V_{N}(\theta) & =\frac{1}{N} \sum_{t=1}^{N} \epsilon(t, \theta)^{2}
\end{aligned}
$$

If $M(\theta)$ is full-order and under reasonable conditions (see below), then $\hat{\theta}_{N}$ will be a consistent estimate of $\theta_{0}$ [1]. In other words, the asymptotic identification criterion, i.e. $\arg \min _{\theta} \bar{E}\left[\epsilon(t, \theta)^{2}\right]$ where the operator $\bar{E}[x(t)]=\lim _{N \rightarrow+\infty} \frac{1}{N} \sum_{t=1}^{N} E[x(t)]$ is defined for quasistationary signals $x(t)[1]$ and $E[x(t)]$ is the expectation of $x(t)$, has an unique solution and is equal to $\theta_{0}$. The first condition is the global identifiability of $M(\theta)$, verified for MISO FIR structure [1]. Therefore, the consistency is equivalent to the second condition which is the data informativity w.r.t. $M(\theta)$. This second condition will be studied in the next section for the considered model structure, i.e. MISO FIR in open loop. 


\section{Data informativity for MISO FIR systems}

For MISO FIR systems in open-loop, the predictor in (2.5) only depends on the input $u(t)$. Then the data informativity defined in [2] is equivalent to the input informativity.

\section{Definition 3.1}

(Informative input for MISO FIR in open loop) The input vector $u(t)$ is said informative w.r.t. $M(\theta)$ if, for any $\theta^{\prime}$ and $\theta^{\prime \prime}$ in $\mathbb{R}^{p m}$, we have:

$$
\bar{E}\left\{\left[G\left(z, \theta^{\prime}\right)-G\left(z, \theta^{\prime \prime}\right)\right] u(t)\right\}^{2}=0 \Rightarrow \theta^{\prime}=\theta^{\prime \prime}
$$

Using (2.5), we have that

$$
\begin{aligned}
& \bar{E}\left\{\left[G\left(z, \theta^{\prime}\right)-G\left(z, \theta^{\prime \prime}\right)\right] u(t)\right\}^{2}=0 \\
\Leftrightarrow & \Delta \theta^{T} \underbrace{\bar{E}\left[\phi_{u}(t) \phi_{u}^{T}(t)\right]}_{R_{u}} \Delta \theta=0
\end{aligned}
$$

where $\Delta \theta=\theta^{\prime}-\theta^{\prime \prime}$. Thus, we have the following theorem:

\section{Theorem 3.1}

The input vector $u(t)$ is informative with respect to $M(\theta)$, if and only if, $R_{u}$ is strictly positive definite.

Proof: The matrix $R_{u}=\bar{E}\left[\phi_{u}(t) \phi_{u}^{T}(t)\right]$ is at least semi-definite positive. Therefore the proof is straightforward by combining Definition 3.1 and the equivalence (3.2).

A regressor $\phi(t)$ satisfying $\bar{E}\left[\phi(t) \phi^{T}(t)\right]>0$ is called persistently exciting (PE) or we say that we have the persistency of this regressor. Therefore, in this case, the persistency of $\phi_{u}(t)$ is equivalent to the consistency of $\hat{\theta}_{N}$. Thus, we will develop conditions on $u(t)$ to obtain the persistency of $\phi_{u}(t)$ (and so the consistency). Then, the problem considered in this paper is the following one:

\section{Problem 3.1}

Find necessary and sufficient conditions on $u(t)$ such that the regressor $\phi_{u}(t)$ in (2.4) is PE.

For scalar input $(p=1)$, there are simple necessary and sufficient conditions for the persistency of $\phi_{u}(t)[2]$.

\section{Lemma 3.1}

Consider the MISO FIR structure of the same order $m$ in (2.2) in open-loop with $p=1$. The regressor in (2.4) is $P E$ if and only if there are at least $m$ different frequencies in the interval ]$-\pi, \pi]$ at which the PSD of $u(t)$ is nonzero.

Proof: See [2] for the detailed proof.

For MISO FIR model structure, the Toeplitz matrix $R_{u}$ in (3.2) is constructed as follows $R_{u}=\left(R_{u_{i} u_{f}}\right)_{(i, f) \in \llbracket 1, p \rrbracket^{2}}$ with $R_{u_{i} u_{f}}=\bar{E}\left[\phi_{u_{i}}(t) \phi_{u_{f}}(t)^{T}\right] \stackrel{\text { if } \stackrel{i=f}{=}}{=} R_{u_{i}}$. 
From this matrix construction, we can extract first conditions on each scalar input for the persistency of $\phi_{u}(t)$ as described in the next theorem:

\section{Theorem 3.2}

Consider the MISO FIR structure of the same order $m$ in (2.2) in open-loop and its derived regressor $\phi_{u}(t)$ in (2.4). If the matrix $R_{u}=\bar{E}\left[\phi_{u}(t) \phi_{u}^{T}(t)\right]$ is strictly positive definite, then for each scalar signal $u_{i}(t)$, there are $m$ different frequencies in the interval $\left.]-\pi, \pi\right]$ at which its $P S D$ is nonzero.

Proof: Assume that $R_{u}$ in (3.2) is strictly positive definite. Hence, all square block matrices $R_{u_{i}}$ must be strictly positive definite. Since $R_{u_{i}}=\bar{E}\left[\phi_{u_{i}}(t) \phi_{u_{i}}(t)^{T}\right]$, all regressors $\phi_{u_{i}}(t)$ must be PE. The proof is obtained by using Lemma 3.1 for $i=1, \cdots, p$.

Theorem 3.2 gives a necessary condition on each scalar input $u_{i}(t)$ : there must be $m$ different frequencies in the interval $]-\pi, \pi]$ at which its power spectrum density (PSD) is nonzero. Contrary to the SISO case [2], this richness condition is not sufficient for the persistency. Indeed, linear dependency between the regressors $\phi_{u_{i}}(t)$ could yield to a singular $R_{u}$. Theorem 3.2 does not answer Problem 3.1, but it gives a first constraint for the choice of $u(t)$.

A necessary and sufficient condition for the persistency of (2.4) is the positive-definiteness of the matrix $R_{u}$ in (3.2), answering Problem 3.1. However this criterion becomes highly complex to verify by hands when the number of inputs and parameters per transfer function increase. Let us therefore find a more effective condition. For this purpose, we will from now onwards consider the case of multisine excitation.

When $\phi_{u}(t)$ is PE, for any $\alpha \in \mathbb{R}^{p m}$, we have $\bar{E}\left[\phi_{u}(t)^{T} \alpha\right]^{2}=0 \Leftrightarrow \alpha=0$. However the quantity $\bar{E}\left[\phi_{u}(t)^{T} \alpha\right]^{2}$ is the power of the multisine signal $\phi_{u}(t)^{T} \alpha$. Therefore, as the power of the multisine is equal to 0 if and only if this signal is identically equal to 0 , we have $\bar{E}\left[\phi_{u}(t)^{T} \alpha\right]^{2}=$ $0 \Leftrightarrow \phi_{u}(t)^{T} \alpha=0 \forall t$. Therefore $\phi_{u}(t)$ is PE if and only if $\phi_{u}(t)^{T} \alpha=0 \forall t \Leftrightarrow \alpha=0$. Denote $\mathcal{U}$ the set formed by all signals of $\phi_{u}$, i.e. the set $\mathcal{U}=\left\{u_{1}(\bullet-1), \cdots, u_{1}(\bullet-m), \cdots, u_{p}(\bullet-\right.$ $\left.1), \cdots, u_{p}(\bullet-m)\right\}$.

\section{Definition 3.2}

[4] A set of real-valued signals $\left\{x_{1}, \cdots, x_{n}\right\}$ is said to be linearly independent in the signal space if, for all $\left(\lambda_{1}, \cdots, \lambda_{n}\right) \in \mathbb{R}^{n}$, we have

$$
\lambda_{1} x_{1}(t)+\cdots+\lambda_{n} x_{n}(t)=0 \forall t \Leftrightarrow \lambda_{1}=\cdots=\lambda_{n}=0
$$

Therefore, in the MISO FIR case in open loop, a necessary and sufficient condition to get the persistency of $\phi_{u}(t)$ is the linear independence of the signal set $\mathcal{U}$ as shown above. Problem 3.1 can be therefore reformulated as follows:

\section{Problem 3.2}

Find sufficient conditions on $u(t)$ such that $\mathcal{U}$ is linearly independent in the signal space, i.e. for all real scalars $\lambda_{i k}$, the following statement is true:

$$
\sum_{k=1}^{m} \sum_{i=1}^{p} \lambda_{i k} u_{i}(t-k)=0 \quad \forall t \Leftrightarrow \text { all } \lambda_{i k}=0
$$


For further references, let us order the scalars $\lambda_{i k}$ in two different ways:

$$
\Lambda=\left(\begin{array}{c}
\Lambda_{1} \\
\vdots \\
\Lambda_{m}
\end{array}\right) \text { with } \Lambda_{k}=\left(\begin{array}{c}
\lambda_{1 k} \\
\vdots \\
\lambda_{p k}
\end{array}\right)
$$

and

$$
L=\left(\begin{array}{c}
L_{1} \\
\vdots \\
L_{p}
\end{array}\right) \text { with } L_{i}=\left(\begin{array}{c}
\lambda_{i 1} \\
\vdots \\
\lambda_{i m}
\end{array}\right)
$$

\section{Persistency for the multisine case}

In the sequel, the index $i \in \llbracket 1, p \rrbracket$ describes the scalar inputs $u_{i}(t), k \in \llbracket 1, m \rrbracket$ the delays $z^{-k}$ and $h \in \llbracket 1, n \rrbracket$ the sinusoid frequencies $\omega_{h}$.

\subsection{General framework for the multisine case}

As already mentioned, we consider the case where all $u_{i}(t)$ are multisine. We have deduced one necessary condition on all $u_{i}(t)$ in Theorem 3.2. As a sinusoid has 2 frequencies in its PSD, this condition becomes: each $u_{i}(t)$ must contain at least $m / 2$ sinusoids at different frequencies. This will be assumed true in the sequel.

Consider $n \geq m / 2$ sinusoids at different frequencies, denoted $\omega_{1}, \cdots, \omega_{h}, \cdots, \omega_{n}$ with $h \in$ $\llbracket 1, n \rrbracket$. For the sake of simplicity, we will exclude the frequencies $d \pi$ with $d$ an integer. The number $n$ is the total number of sinusoids at different frequencies in the input vector $u(t)$. Then, each input $u_{i}(t)$ is in the following form

$$
u_{i}(t)=\sum_{h=1}^{n} a_{i h} \cos \left(\omega_{h} t+\psi_{i h}\right)
$$

where all $a_{i h}$ are amplitudes and all $\psi_{i h}$ are phase shifts. If $u_{i}(t)$ is not excited by the sinusoid at $\omega_{h}$, then $a_{i h}=0$. With Euler formula, (4.1) can be written in the complex form:

$$
u_{i}(t)=\frac{1}{2}\left(\sum_{h=1}^{n} \bar{A}_{i h} e^{j \omega_{h} t}+\sum_{h=1}^{n} \bar{A}_{i h}^{*} e^{-j \omega_{h} t}\right)
$$

where $\bar{A}_{i h}=a_{i h} e^{j \psi_{i h}}$ is a phasor. By injecting (4.2) in the left hand side (lhs) of (3.3), we get:

$$
\sum_{h=1}^{n} \Upsilon_{h} e^{j \omega_{h} t}+\sum_{h=1}^{n} \Upsilon_{h}^{*} e^{-j \omega_{h} t}=0 \quad \forall t
$$

with $\Upsilon_{h}=\sum_{k=1}^{m} \sum_{i=1}^{p} \lambda_{i k} \bar{A}_{i h} e^{-j k \omega_{h}}$.

For each complex scalar $\Upsilon_{h}$, denote $\Upsilon_{h}=\alpha_{h}+j \beta_{h}$ where $\alpha_{h}$ and $\beta_{h}$ are real scalars. The equality (4.3) leads to

$$
\sum_{h=1}^{n} \alpha_{h} \cos \left(\omega_{h} t\right)-\sum_{h=1}^{n} \beta_{h} \sin \left(\omega_{h} t\right)=0 \quad \forall t
$$


As all frequencies $\omega_{h}$ are different between them and different from $0, \pi$ and $-\pi$, it is well known that the signal set $\left\{\cos \left(\omega_{1} \bullet\right), \cdots, \cos \left(\omega_{n} \bullet\right)\right\} \cup\left\{\sin \left(\omega_{1} \bullet\right), \cdots, \sin \left(\omega_{n} \bullet\right)\right\}$ is linearly independent in the signal space [5]. Therefore, from Definition 3.2 applied to (4.4), all $\alpha_{h}=0$ and all $\beta_{h}=0$. Then, for the lhs of (3.3) to hold, we need all $\Upsilon_{h}=0$ and $\Upsilon_{h}^{*}=0$. Regrouping all the equations $\Upsilon_{h}=0$ and $\Upsilon_{h}^{*}=0$ in an equation system leads to

$$
\sum_{k=1}^{m} \underbrace{\left(\begin{array}{c}
\sum_{i=1}^{p} \lambda_{i k} \bar{A}_{i 1} e^{-j k \omega_{1}} \\
\sum_{i=1}^{p} \lambda_{i k} \bar{A}_{i 1}^{*} e^{j k \omega_{1}} \\
\vdots \\
\sum_{i=1}^{p} \lambda_{i k} \bar{A}_{i n} e^{-j k \omega_{n}} \\
\sum_{i=1}^{p} \lambda_{i k} \bar{A}_{i n}^{*} e^{j k \omega_{n}}
\end{array}\right)}_{W_{k}}=\left(\begin{array}{c}
0 \\
0 \\
\vdots \\
0 \\
0
\end{array}\right)
$$

For each term $W_{k}$ of (4.5), all $\lambda_{i k}$ of (4.5) can be isolated:

$$
W_{k}=\underbrace{\left(\begin{array}{ccc}
\bar{A}_{11} e^{-j k \omega_{1}} & \ldots & \bar{A}_{p 1} e^{-j k \omega_{1}} \\
\bar{A}_{11}^{*} e^{j k \omega_{1}} & \ldots & \bar{A}_{p 1}^{*} e^{j k \omega_{1}} \\
\vdots & \vdots & \vdots \\
\bar{A}_{1 n} e^{-j k \omega_{n}} & \cdots & \bar{A}_{p n} e^{-j k \omega_{n}} \\
\bar{A}_{1 n}^{*} e^{j k \omega_{n}} & \cdots & \bar{A}_{p n}^{*} e^{j k \omega_{n}}
\end{array}\right)}_{\mathcal{L}_{k}} \Lambda_{k}
$$

where $\Lambda_{k}$ is defined in (3.4).

Therefore with (4.6) $\forall k \in \llbracket 1, m \rrbracket$, the system (4.5) can be written in the following row-block matrix equation:

$$
\underbrace{\left(\begin{array}{lll}
\mathcal{L}_{1} & \cdots & \mathcal{L}_{m}
\end{array}\right)}_{\mathcal{L}} \Lambda=0
$$

where $\mathcal{L}$ is a $2 n \times p m$ row-block matrix. We can therefore deduce the following necessary and sufficient condition to solve Problem 3.2 with multisine excitation:

\section{Theorem 4.1}

The regressor in (2.4) is $P E$, if and only if, the matrix $\mathcal{L}$ in (4.7) is full-column rank.

Proof: The regressor in (2.4) is $\mathrm{PE}$ if and only if the signals in $\mathcal{U}$ are linearly independent. Since the lhs of (3.3) can be rewritten as in (4.7), the latter holds if and only if $\Lambda=0$ is the only solution of the linear matrix equality (4.7) and a necessary and sufficient condition for this is indeed that $\mathcal{L}$ is full column rank.

We have deduced a simple necessary and sufficient condition for the persistency of $\phi_{u}(t)$ for multisine excitation. This criterion is easy to verify and only depends on the chosen amplitudes, frequencies, phase shifts and on the orders of the FIR filters $G_{i}(z, \theta)$. But Theorem 4.1 does not allow us to directly choose the multisine number $n$, the amplitudes and the phase shifts which ensure that $\mathcal{L}$ is full-column rank. In other words, our multisine choice can only be validated a posteriori, with the rank verification of $\mathcal{L}$. It would be interesting to know some additional conditions for this choice before computing the matrix $\mathcal{L}$. For that, each $\mathcal{L}_{k}$ in $(4.6)$ can be written as follows:

$$
\mathcal{L}_{k}=E^{k} \bar{A}
$$


where $E=\operatorname{diag}\left(e^{-\omega_{1}}, e^{\omega_{1}}, \cdots, e^{-\omega_{n}}, e^{\omega_{n}}\right)$ is a $2 n \times 2 n$ diagonal matrix, $E^{k}=\underbrace{E \times \cdots \times E}_{k \text { times }}$ and $\bar{A}$ is:

$$
\bar{A}=\left(\begin{array}{ccc}
\bar{A}_{11} & \cdots & \bar{A}_{p 1} \\
\bar{A}_{11}^{*} & \cdots & \bar{A}_{p 1}^{*} \\
\vdots & \vdots & \vdots \\
\bar{A}_{1 n} & \cdots & \bar{A}_{p n} \\
\bar{A}_{1 n}^{*} & \cdots & \bar{A}_{p n}^{*}
\end{array}\right)
$$

The matrix $\bar{A}$ in (4.9) is called the phasor matrix. Therefore, with (4.8), the matrix $\mathcal{L}$ becomes:

$$
\mathcal{L}=\left(\begin{array}{lll}
E \bar{A} & \cdots & E^{m} \bar{A}
\end{array}\right)
$$

One can remark that $\mathcal{L}$ is constructed such that each block is a delayed version of the phasors of all $u_{i}(t)$ and the blocks are put in ascending order of delays. The matrix $\mathcal{L}$ can be written in the following block-form:

$$
\mathcal{L}=\underbrace{\left(E \cdots E^{m}\right)}_{\mathcal{E}} \underbrace{\operatorname{diag}(\bar{A}, \cdots, \bar{A})}_{\mathcal{A}}
$$

where $\mathcal{E}$ is a $2 n \times 2 n m$ row block matrix and $\mathcal{A}$ is a $2 n m \times m p$ diagonal block matrix composed of $m$ matrices $\bar{A}$. From $\mathcal{L}=\mathcal{E} \mathcal{A}$ in (4.11), we can deduce additional conditions on the sinusoid number $n$ and the choices of the multisine amplitudes and phase shifts (or the phasor matrix), given in the next theorem

\section{Theorem 4.2}

If the matrix $\mathcal{L}$ is full-column rank, then it is necessary that:

- (i) $\bar{A}$ in (4.9) is full-column rank.

- (ii) $n \geq p m / 2$.

Proof: Point (ii) : Since $\mathcal{L}$ is full-column rank, its number of rows must be greater or equal to its number of columns. Therefore $2 n \geq p m$, i.e. $n \geq p m / 2$.

Point $(i)$ : Assume that $\mathcal{L}=\mathcal{E} \mathcal{A}$ is full-column rank. Therefore the matrix $\mathcal{A}$ is full-column rank. It is a block-diagonal matrix composed by $m$ matrices $\bar{A}$. Then due to its construction, $\mathcal{A}$ is full-column rank, if and only if, $\bar{A}$ is full-column rank. Therefore $\bar{A}$ must be full-column rank.

\section{Remark 4.1}

The conditions ( $i$ ) and (ii) of Theorem 4.2 are not sufficient for Theorem 4.1. However they give more insight into the multisine choice that has to be done to get the persistency.

\section{Remark 4.2}

Combining Theorem 3.2 with the point (ii) of Theorem 4.2 gives the least necessary sinusoid number for the informativity, i.e. we just need that number of sinusoids. Then, the matrix criterion of Theorem 4.1 only depends on the FIR orders, the frequencies and the phasors.

We are going to study particular multisine choices which always provide the persistency of $\phi_{u}(t)$. By permuting the variables in $\Lambda$ and the columns of the matrix equation $\mathcal{L}$, the matrix 
system in (4.7) is equivalent to the following one:

$$
\underbrace{\left(\begin{array}{lll}
Q_{1} P & \cdots & Q_{p} P
\end{array}\right)}_{\mathcal{Q}} L=0
$$

$$
\begin{aligned}
& \text { with } Q_{i}=\operatorname{diag}\left(\bar{A}_{i 1}, \bar{A}_{i 1}^{*}, \cdots, \bar{A}_{i n}, \bar{A}_{i n}^{*}\right) \\
& \text { and } P=\left(\begin{array}{ccc}
e^{-j \omega_{1}} & \cdots & e^{-j m \omega_{1}} \\
e^{j \omega_{1}} & \cdots & e^{j m \omega_{1}} \\
\vdots & \cdots & \vdots \\
e^{-j \omega_{n}} & \cdots & e^{-j m \omega_{n}} \\
e^{j \omega_{n}} & \cdots & e^{j m \omega_{n}}
\end{array}\right)
\end{aligned}
$$

where $L$ is defined in (3.5) and $\mathcal{Q}$ is a $2 n \times m p$ row-block matrix. Remark that $\mathcal{Q}$ is constructed such that each block contains all delayed versions of the phasors of one input $u_{i}(t)$ and the blocks are put in ascending order of inputs.

\section{Corollary 4.1}

The regressor in (2.4) is $P E$, if and only if, the matrix $\mathcal{Q}$ in (4.12) is full-column rank.

Proof: Straightforward by observing that $\mathcal{L}$ and $\mathcal{Q}$ are equivalent and by using Theorem 4.1.

The form in (4.12) will be useful for the next paragraph, dealing with particular cases of multisine excitation. But before that, we give an useful lemma for the sequel.

\section{Lemma 4.1}

If $u_{i}$ contains at least $m / 2$ sinusoids of distinct frequencies (not equal to $d \pi, d$ integer), then $Q_{i} P$ is full-column rank.

Proof: Straightforward consequence of Lemma 3.1 and Corollary 4.1 since $\mathcal{Q}$ reduces to $Q_{i} P$ when $u=u_{i}$ (SISO case).

\subsection{Particular and practical results for the multisine case}

In the two next corollaries, we present particular multisine choices, where $\phi_{u}(t)$ is $\mathrm{PE}$.

\section{Corollary 4.2}

If, in the choices of the sinusoids,

- (a) $u_{1}(t)$ is excited by at least $m / 2$ different sinusoids,

- (b) $\forall i \in \llbracket 2, p \rrbracket, u_{i}(t)$ contains the the same sinusoids as $u_{i-1}(t)$ (the same frequencies) but also contains at least $m / 2$ more sinusoid w.r.t. $u_{i-1}(t)$,

then the regressor $\phi_{u}(t)$ is $P E$.

Proof: We will prove that $\mathcal{Q}$ is full-column rank (see Corollary 4.1). Denote $r_{i}$ the total number of sinusoids in $u_{i}(t)$ and $n_{i}$ the additional number of sinusoids in $u_{i}(t)$ w.r.t. $u_{i-1}(t)$, i.e. $r_{i}=n_{i}+r_{i-1}$, $\forall i \in \llbracket 2, p \rrbracket$. From $(a)$ and $(b)$, we have $r_{i} \geq m / 2 \forall i \in \llbracket 1, p \rrbracket$ and $n_{i} \geq m / 2 \forall i \in \llbracket 2, p \rrbracket$. 
With this input choice, each input $u_{i}(t)$ is expressed as in (4.1) with $a_{i h} \neq 0 \quad \forall h \leq r_{i}$ and $a_{i h}=0$ $\forall h>r_{i}$. Thus we have the same properties on the phasors: $\bar{A}_{i h} \neq 0 \quad \forall h \leq r_{i}$ and $\bar{A}_{i h}=0 \quad \forall h>r_{i}$ in (4.9). Hence, the matrix $Q$ has an block-upper-triangular form given by

$$
Q=\left(\begin{array}{cccc}
Q_{11} & Q_{12} & \cdots & Q_{1 p} \\
\mathbf{0}_{21} & Q_{22} & \cdots & Q_{2 p} \\
\vdots & \vdots & \ddots & \vdots \\
\mathbf{0}_{p 1} & \mathbf{0}_{p 2} & \cdots & Q_{p p}
\end{array}\right)
$$

where $\mathbf{0}_{s i}$ is the $2 n_{s} \times m$ zero matrix with $s \in \llbracket i+1, p-1 \rrbracket$ and $Q$ has a size $2 n_{i} \times m$. The matrix $Q$ is full-column rank if and only if all $Q_{i i}$ are all full-column rank which is the case as $\mathcal{Q}$ reduces to $Q_{i i}$ when $u=u_{i}$ (SISO case) and each $u_{i}$ is sufficiently rich (since $n_{i} \geq m / 2 \forall i \in \llbracket 1, p \rrbracket$ ).

\section{Remark 4.3}

In Corollary 4.2, we consider $u_{1}(t)$ for condition $(a)$ and the other inputs in ascending order for $(b)$. This result is still true by considering any input $u_{i}(t)$ for $(a)$ and the other inputs in any order for $(b)$.

\section{Corollary 4.3}

If each input $u_{i}(t)$ is excited with at least $m / 2$ different sinusoids which are different from the ones of the other inputs (no common excitation frequencies between the inputs), then the regressor $\phi_{u}(t)$ is $P E$.

Proof: Same proof as Corollary 4.2, as the obtained matrix $\mathcal{Q}$ here is block-diagonal.

The experiment choice of Corollary 4.3 is the most simple one, as it considers the least number of frequencies to use for each scalar input, but not necessarily the optimal one for all identification problems. For optimal experiment design [6], with multisine as in [7] and [8], it might be wise to consider the general framework in section 4.1, as we will have more flexibility for the optimal input design than an experiment choice like the one in Corollary 4.3.

\subsection{Synthesis of the multisine excitation results}

In this section, we have developed a sufficient condition for the consistency of the identification of MISO FIR structure in open loop with multisine: Theorem 4.1. It can be used, before the experiment, to check if the chosen multisine input will lead to a consistent identification, by simply checking the rank of $\mathcal{L}$ in (4.7). However, with Theorem 4.1 we cannot make an accurate decision in the input choice which ensures that $\mathcal{L}$ is full-column rank. One idea is to choose randomly the amplitudes and phase shifts. For some random draws, $\mathcal{L}$ will be full-column rank, but this approach is not wise.

That is why we have developed also additional conditions to guide better the user in its choices of amplitudes, phase shifts and sinusoid number. They are described in Theorems 3.2 and 4.2. Theorem 3.2 combined with (ii) of Theorem 4.2 gives an useful condition on the total number of sinusoids $(n)$ and per input (number of nonzero phasors/amplitudes per input). The point $(i)$ can be used as a first verification step (rank verification of $\bar{A}$ ) before verifying the rank of $\mathcal{L}$. As they are not sufficient, it is not possible to avoid the rank verification of $\mathcal{L}$ in the general case. 
This is the reason why we studied two particular cases in Corollaries 4.2 and 4.3. Indeed these particular cases lead to the persistency of the regressor $\phi_{u}(t)$ regardless of the choices of the phase shifts and the amplitudes. Note that there is no needed rank verification in both cases.

\section{$5 \quad$ Numerical example}

We consider a MISO FIR system $\mathcal{S}$ described in (2.1) with $p=2$ inputs, $m=2$ parameters per $G_{0_{i}}$ :

$$
G_{0_{1}}(z)=0.22 z^{-1}-0.63 z^{-2} \text { and } G_{0_{2}}(z)=z^{-1}+0.95 z^{-2}
$$

For the inputs, we choose:

$$
\left\{\begin{array}{l}
u_{1}(t)=a_{11} \cos \left(\omega_{1} t+\phi_{11}\right)+a_{12} \cos \left(\omega_{2} t+\phi_{12}\right) \\
u_{2}(t)=a_{21} \cos \left(\omega_{1} t+\phi_{21}\right)+a_{22} \cos \left(\omega_{2} t+\phi_{22}\right)
\end{array}\right.
$$

with $\omega_{1}=0.1$ and $\omega_{2}=0.25$. We will propose several input choices to test the conditions developed in Section 4.1. For each choice, we identify $G(z, \theta)$ with $N$ input-output data from $\mathcal{S}$ and we compute the rank of $\mathcal{L}$ in (4.7) and $\bar{A}$ in (4.9).

In case of consistency, $\operatorname{rank}(\bar{A})=2$ and $\operatorname{rank}(\mathcal{L})=4$. For FIR systems, the modeling error $\hat{\theta}_{N}-\theta_{0}$ of the computed parameters is normally distributed around 0 [1]. Thus the relative error $\left(\hat{\theta}_{N}-\theta_{0}\right) / \theta_{0}$ is normally distributed around $0 \%$. We consider $N=10000$ and $\sigma_{0}^{2}=0.001$ for small variance of the relative error to facilitate the consistency verification. The notation $\operatorname{err}\left(b_{i k}\right)$ refers to the relative error between the computed parameter $b_{i k}$ and the true parameter $\beta_{i k}$. The results of the simulation for each case are given in Table 1.

First we verify that both conditions developed in Theorem 4.2 are necessary by considering 2 cases when each of both conditions is not satisfied.

Case 1: We consider a case where $\bar{A}$ degenerates. Consider the amplitudes and phase-shifts such that $u_{1}=2 u_{2}$ :

$$
\begin{aligned}
& \text { - } a_{11}=2, \quad a_{12}=2, \quad a_{21}=1, \quad a_{22}=1 \\
& \text { - } \phi_{11}=0, \quad \phi_{12}=0, \quad \phi_{21}=0, \quad \phi_{22}=0
\end{aligned}
$$

With these values, $\operatorname{rank}(\bar{A})=1$ (not full-column rank, linear dependency of the phasors) and $\operatorname{rank}(\mathcal{L})=2$ (not full-column rank). The non-consistency is verified in simulation, in the Table 1, as the relative errors are not close to $0 \%$.

Case 2: Now, we consider a case where the condition (ii) of Theorem 4.2 is not respected by using only one sinusoid per input:

$$
\begin{aligned}
& \text { - } a_{11}=1, a_{12}=0, a_{21}=1, \quad a_{22}=0 \\
& \text { - } \phi_{11}=0.5, \quad \phi_{12}=0, \phi_{21}=-0.7, \quad \phi_{22}=0
\end{aligned}
$$

We have $\operatorname{rank}(\bar{A})=2$ (full-column $\operatorname{rank})$ and $\operatorname{rank}(\mathcal{L})=2$ (not full-column rank). Indeed, here both inputs are excited with one sinusoid at the the same frequency $\omega_{1}$, i.e. $n=1$ : the necessary 
condition (ii) of Theorem 4.2 is violated as $p m / 2=2$ and $n=1$. Therefore $\mathcal{L}$ degenerates illustrated with the high relative error in the Table 1.

However the conditions in Theorem 4.2 are not sufficient. We can construct cases when they are both satisfied while the matrix $\mathcal{L}$ in (4.7) degenerates:

Case 3: Consider these following values:

- $a_{11}=1, a_{12}=1, a_{21}=3, a_{22}=3$

- $\phi_{11}=-\omega_{1}, \phi_{12}=-\omega_{2}, \phi_{21}=0, \phi_{22}=0$

With these values, $\operatorname{rank}(\bar{A})=2$ (full-column rank) and $n=2=p m / 2$. Therefore, both conditions in Theorem 4.2 are respected. However we have $\operatorname{rank}(\mathcal{L})=3$ (not full-column rank). This highlights that $(i)$ and $(i i)$ are not sufficient.

Indeed, by looking at the chosen phase shifts, we have $u_{2}(t-2)=3 u_{1}(t-1)$ for all $t$. Therefore the consistency is not reached for $b_{11}$ and $b_{22}$. As $u_{1}(\bullet-2)$ and $u_{2}(\bullet-1)$ are not correlated with another signal in $\phi_{u}$, then the consistency is obtained for $b_{12}$ and $b_{21}$. The results from simulation in the Table 1 meet our remarks.

Sometimes, with proper values of amplitudes and phase-shifts, the consistency is obtained.

Case 4: For instance with the following values

- $a_{11}=1, a_{12}=1, a_{21}=1, a_{22}=1$

- $\phi_{11}=-0.4, \phi_{12}=0.3, \phi_{21}=0, \phi_{22}=-0.7$

we have $\operatorname{rank}(\mathcal{L})=4$ (full-column rank). Therefore, from Theorem 4.1, the consistency is reached, as illustrated by the values of simulation in Table 1 . This case is interesting as it shows that we can identify $\mathcal{S}$ by using the the same frequencies in all scalar inputs.

But our choice validation is still verified a posteriori. For some particular cases, the consistency will always be reached regardless of the amplitude and phase shift choices. This is the case of experiment choice respecting Corollary 4.2 or Corollary 4.3, illustrated for Corollary 4.2 in the next case.

Case 5: The experiment choice is the following one:

- $a_{11}=1, \quad a_{12}=0, a_{21}=1, a_{22}=1$

- $\phi_{11}=-0.4, \phi_{12}=0.3, \phi_{21}=0, \phi_{22}=-0.7$

Here, $\operatorname{rank}(\mathcal{L})=4$ (full-column rank). Indeed we are in the case of Corollary 4.2 , verified in simulation in Table 1. 
Table 1: Parameter relative errors for all cases.

\begin{tabular}{|c|c|c|c|c|}
\hline & $\operatorname{err}\left(b_{11}\right)$ & $\operatorname{err}\left(b_{12}\right)$ & $\operatorname{err}\left(b_{21}\right)$ & $\operatorname{err}\left(b_{22}\right)$ \\
\hline Case 1 & $188.37 \%$ & $82.29 \%$ & $74.53 \%$ & $109.35 \%$ \\
\hline Case 2 & $182.71 \%$ & $71.32 \%$ & $28.17 \%$ & $26.97 \%$ \\
\hline Case 3 & $192.41 \%$ & $0.89 \%$ & $0.42 \%$ & $15.80 \%$ \\
\hline Case 4 & $0.9 \%$ & $0.32 \%$ & $0.19 \%$ & $0.22 \%$ \\
\hline Case 5 & $1.22 \%$ & $0.05 \%$ & $0.09 \%$ & $1.04 \%$ \\
\hline
\end{tabular}

\section{Conclusions}

This paper considers the problem of data informativity for MISO FIR systems in open-loop. In this case, the informativity is equivalent to the persistency of the regressor derived form the MISO FIR model structure. This persistency is equivalent to the linear independence of the signals composing this regressor. Based on this consideration, we developed a simple necessary and sufficient condition to for the regressor persistency (and so for the consistency of the parameter vector estimation). We also give necessary conditions and particular cases to guide the reader in its input vector choice. A numerical example has been proposed to verify several results developed in this paper. A lot of perspectives are ongoing. We want to extend this study in closed-loop and also for other model structures such as Box-Jenkins, ARMAX, ARX and Ouput-Error.

\section{References}

[1] L. Ljung, System Identification: Theory for User, 1987.

[2] M. Gevers, A. S. Bazanella, X. Bombois, and L. Miskovic, "Identification and the information matrix: How to get just sufficiently rich?" IEEE Transactions on Automatic Control, vol. 54, no. 12 , pp. 2828-2840, Dec 2009.

[3] T. Soderstrom and P. Stoica, System Identification. Prentice Hall, 1989.

[4] G. Cariolaro, Unified signal theory. Springer Science \& Business Media, 2011.

[5] A. Setniker, "Linear independence in function spaces," Honors Senior Theses/Projects, pp. 4-6, 2013.

[6] X. Bombois, G. Scorletti, M. Gevers, P. M. Van den Hof, and R. Hildebrand, "Least costly identification experiment for control," Automatica, vol. 42, no. 10, pp. 1651-1662, 2006.

[7] X. Bombois, M. Barenthin, and P. M. Van den Hof, "Finite-time experiment design with multisines," IFAC Proceedings Volumes, vol. 41, no. 2, pp. 11 445-11 450, 2008.

[8] H. Jansson, "Experiment design with applications in identification for control," Ph.D. dissertation, Royal Institute of Technology (KTH), 2004. 